\title{
Intervention study on Chinese medicine Tongxinluo for cardiomyopathy in diabetic rats
}

\author{
Xiaoyan Zhang, Xiaomei Wang*, Fenghua Li, Xiaoli Cui, Xinyu Liu, Lichao Hui, Shuo \\ Wang, Na Song and Nannan Li
}

The Affiliated First Hospital of Liaoning Medical University, Jinzhou 121001, China.

Accepted 19 November, 2012

\begin{abstract}
The aim of this study was to observe the effect of early application of the Chinese Medicine Tongxinluo on cardiomyopathy in diabetic rats. 36 rats were randomly divided into normal control group, diabetic group and Tongxinluo (TXL) group. Streptozotocin was used for preparation of diabetic model of Sprague Dawley (SD) rats. Tongxinluo powder (1.43 $\mathrm{g}$ of crude drug/g dry powder) was dissolved in water and used for gavage at a dosage of $0.3 \mathrm{~g} \mathrm{~kg}^{-1} \mathrm{day}^{-1}$. Blood glucose was detected 8 weeks later; left ventricular systolic pressure and left ventricular end diastolic pressure, maximum rise and fall rate of left ventricular pressure $( \pm \mathrm{dp} / \mathrm{dt} \max )$ were determined; myocardial pathological changes were observed under light microscopy and electron microscopy after Hematoxylin and eosin (HE) staining. \pm $\mathrm{dp} / \mathrm{dt}$ max in the diabetic rat group decreased, but $\pm \mathrm{dp} / \mathrm{dt}$ max of the Tongxinluo group increased, and the difference was significant $(P<0.05)$. Under light microscope, the myocardium myofibril of the diabetic group was deranged, and myofilament composition was reduced. Electron microscopy showed that cardiac endothelial cells were markedly swollen, and the basilar membrane of capillary vessel showed irregular obvious thickening. The lesions of the Tongxinluo group were mild. Tongxinluo can significantly improve cardiac function of diabetic rats and reduce diabetic cardiomyopathy.
\end{abstract}

Key words: Tongxinluo, diabetic rats, diabetic cardiomyopathy.

\section{INTRODUCTION}

Diabetic cardiomyopathy (DCM) refers to a disease state in which a wide range of structural abnormalities is caused by primary cardiac myocyte injury in patients with diabetes, ultimately leading to left ventricular hypertrophy, diastolic and (or) systolic dysfunction (Hayat et al., 2004), and is one of the major complications of diabetes, and is one of the main reasons leading to the death of patients with diabetes (Laakso, 1999).

Since Rubler et al. (1972) first proposed the diabetic cardiomyopathy, results of clinical, epidemiology, pathology and experimental studies all showed the presence of specific cardiomyopathy, instead of blood supply disorder secondary to coronary. Early diabetic cardiomyopathy may be asymptomatic, as the disease progresses, patients may have angina, arrhythmia and progressive

*Corresponding author. E-mail: xywscn@163.com. Tel: 86-4164197161. cardiac dysfunction, due to the complexity of its causes but it also has its own pathophysiological change, so there are certain difficulties in clinical research and treatment. Therefore, exploration of the pathogenesis of diabetic cardiomyopathy and taking effective measures to control the occurrence and development of diabetic cardiomyopathy are of important significance to improve the quality of life of patients with diabetes and reduce its clinical mortality.

Diabetic cardiomyopathy is of significant influence on the prognosis of diabetic patients, therefore, to strengthen the study on its prevention and control is of great significance for the prevention of diabetic cardiovascular events. Chinese medicine has some advantages in prevention of diabetes and its complications, but there are still many problems in the current study, the focus of most research is still limited to lower blood glucose; studies on diabetic cardiomyopathy is lagging behind and is still limited to the description of the phenomenon, and the study on mechanism is far from in-depth research. 
Traditional Chinese medicine Tongxinluo can significantly improve myocardial ischemia and not only protect the integrity of the structure and function of small blood vessels, and its cardiac microvascular protective effect is more obvious. As a result, we conceptualized that the pathological changes of the Diabetic cardiomyopathy (DCM) are also caused by extensive microvascular change, prevention and treatment of DCM cardiomyopathy with Tongxinluo may have the same effect. This experimental design, with the use of the effect of traditional Chinese medicine Tongxinluo on DCM, is aimed to provide a theoretical basis for the seeking of effective drug treatment for the prevention and treatment of DCM, to make Tongxinluo have a new and broader prospect.

In this study, with the use of Tongxinluo for intervention in streptozotocin (STZ) diabetic rats, the effect of Tongxinluo on structure and function of the myocardium of diabetic rats was observed to ascertain the myocardial protective effect of the drug, so as to provide a theoretical basis for clinical treatment.

\section{MATERIALS AND METHODS}

\section{Reagents}

Streptozotocin (STZ) was purchased from Sigma (USA). The Tongxinluo ultrafine powder provided by Hebei Yiling Pharmaceutical Co., Ltd. (China) is mainly composed by ginseng, leeches, scorpion, eupolyphaga, centipedes, borneol, etc.

\section{Animals and experimental groups}

Male Sprague Dawley (SD) rats, aged 7 to 8 weeks, weight of 180 to $220 \mathrm{~g}$, were provided by Liaoning Medical Experimental Animal Center. The 40 SD rats were randomly divided into two groups: 28 rats were taken and injected with $1 \%$ of STZ which was temporarily prepared with $0.5 \mathrm{mmol} / \mathrm{L}$ sterile citrate-citrate sodium buffer $(\mathrm{pH}$ 4.5 ) by a single injection in the tail vein of the rats at a dosage of 45 $\mathrm{mg} / \mathrm{kg}$; another 12 rats were used as the control group (control, CON) and injected with the same volume of the above buffer. Blood was taken from tail vein $72 \mathrm{~h}$ after the injection for blood glucose determination, rats with blood glucose concentration greater than $16.7 \mathrm{mmol} / \mathrm{L}$ were deemed as a model of diabetes. Four rats with blood glucose lower than this value was discarded, and then the diabetic rats $(n=24)$ were randomly divided into two groups: the diabetic group (DM), and Tongxinluo group (TXL). Rats of each group were reared in the cages of standard conditions, and reared for 8 weeks with standard diet (provided by Liaoning Medical Experimental Animal Center), and the Tongxinluo intervention group was given the Tongxinluo powder (1.43 g of crude drug $/ \mathrm{g}$ dry powder) soluble in water at a dosage of $0.3 \mathrm{~g} \mathrm{~kg}^{-1}$ day ${ }^{-1}$ by gavages. This study was carried out in strict accordance with the recommendations in the Guide for the Care and Use of Laboratory Animals of the National Institutes of Health. The animal use protocol has been reviewed and approved by the Institutional Animal Care and Use Committee (IACUC) of Liaoning Medical University.

\section{Blood glucose determination}

Tail blood was drawn from rats in each group before sacrificed, the glucose oxidase-peroxidase method was used, and the SureStep (steady doubly type) blood glucose monitoring device produced by Plus Johnson \& Johnson and blood glucose test strips were used for blood glucose determination.

\section{Determination of cardiac function}

The rats were anesthetized with $20 \%$ of urethane $(0.5 \mathrm{ml} / \mathrm{kg}$ body weight), catheter was intubated through the left ventricular cavity to the apex, the other end of the catheter was connected to a pressure transducer to record the left ventricular pressure curve (LVP), the LVP electrical signal was input into the Pclab biological signal acquisition and processing system to measure heart rate, maximum rise and fall rate of left ventricular pressure $( \pm \mathrm{dp} / \mathrm{dt} \max )$, left ventricular systolic pressure (LVSP) and Left ventricular end diastolic pressure (LVEDP).

\section{Myocardial paraffin slicing and staining with HE}

After sacrificing the rats in each group, tissue blocks from left ventricle was taken, fixed with $4 \%$ neutral formalin, dehydrated with graded ethanol, embedded in paraffin, and conventionally prepared into myocardial paraffin slices with a slice thickness of $5 \mu \mathrm{m}$, then stained with $\mathrm{HE}$, and the slices were sealed with neutral gum.

\section{Preparation of ultrathin myocardial slices and observation under TEM}

Two rats in each group were randomly selected, anaesthetized with $20 \%$ urethane by intraperitoneal injection, then left ventricular myocardial tissue blocks were taken and cut into tissue blocks of 1 $\mathrm{mm}^{3}$, and the ultra small specimens were placed into $3 \%$ glutaraldehyde fixative for fixation of $2 \mathrm{~h}$, then fixed in $1 \%$ osmium tetroxide for $1 \mathrm{~h}$, and then dehydrated with alcohol and acetone step by step, embedded with epoxy resin, and ultra-thin slices were sliced, with a slice thickness of $50 \mathrm{~nm}$, after double stained with uranium and lead, the myocardial ultrastructural changes were observed with JEOL1200EX TEM scope. 10 electron microscope images of rat heart muscle capillaries $(100 \times 200$ power) were taken from each group. The basement membrane cross-sectional area was the area of interest. 10 points at each capillary were selected for basement membrane thickness measurements. Means were calculated.

\section{Statistical analysis}

The SPSS11.5 statistical software was adopted for data processing and analysis, all measurement data were expressed as mean \pm standard deviation (mean \pm SD), the single factor analysis of variance (SNK method for $q$ test), and $P<0.05$ was for significant difference and $P<0.01$ was for highly significant difference.

\section{RESULTS}

\section{General state of the rats}

At 2 days after injection of STZ, most rats showed diabetic symptoms such as polyuria, polydipsia, polyphagia, slow body weight gain, accompanied by various degree of listlessness, sparse and lackluster fur; four rats with blood glucose lower than $16.7 \mathrm{mmol} / \mathrm{L}$ were 
Table 1 Effect of Tongxinluo on blood glucose and heart function of STZ diabetic rat ( $\left.\bar{x}_{ \pm s}\right)$

\begin{tabular}{lcccccc}
\hline Group & Rat(n) & $\begin{array}{c}\text { blood glucose } \\
(\mathbf{m m o l} / \mathbf{L})\end{array}$ & $\begin{array}{c}\text {-dp/dtmax } \\
(\mathbf{m m H g} / \mathbf{s})\end{array}$ & $\begin{array}{c}\text { +dp/dtmax } \\
(\mathbf{m m H g} / \mathbf{s})\end{array}$ & LVSP & LVEDP \\
\hline CON & 12 & $9.2 \pm 4.56$ & $3688.01 \pm 375.27$ & $3870.54 \pm 386.18$ & $126.66 \pm 7.56$ & $3.74 \pm 0.36$ \\
DM & 10 & $29.5 \pm 2.21$ & $2718.05 \pm 255.90^{*}$ & $2907.27 \pm 148.86^{*}$ & $110.52 \pm 11.03$ & $5.24 \pm 0.42^{* *}$ \\
TXL & 10 & $25.4 \pm 3.25$ & $3000.35 \pm 133.96$ & $3168.36 \pm 166.63$ & $112.60 \pm 11.59^{*}$ & $4.69 \pm 1.05^{\star}$ \\
\hline
\end{tabular}

Note: ${ }^{*} p<0.05^{* *} p<0.01$ verse CON group

discarded, four rats died during feeding, and the general state of the TXL group was better than that of the DM group.

\section{Effect of tongxinluo on blood glucose}

After injection with STZ, blood glucose levels of the rats significantly increased and always fluctuated at a high level during the experiment; when fed for 8 weeks, blood glucose levels $(29.5 \pm 2.21) \mathrm{mmol} / \mathrm{L}$ of rats in the $\mathrm{DM}$ group were significantly higher than that $(9.2 \pm 4.56)$ $\mathrm{mmol} / \mathrm{L}$ in the CON group, blood glucose $(25.4 \pm 3.25)$ $\mathrm{mmol} / \mathrm{L}$ of the TXL group was at much higher levels, and showed no significant difference as compared with that of the DM group (Table 1).

\section{Effect of tongxinluo on heart function of STZ diabetic rat}

Heart function of the experimental rats can be determined by measuring left ventricular $\pm \mathrm{dp} / \mathrm{dt} \max$, LVSP and LVEDP, and changes in $\pm \mathrm{dp} / \mathrm{dt}$ max, LVSP and LVEDP, respectively reflected systolic dysfunction and diastolic dysfunction. When compared with the CON group at duration of 8 weeks, the $\pm \mathrm{dp} / \mathrm{dt}$ max of the DM group reduced for all rats, and the difference was significant; the LVEDP increased more significantly, and the difference was highly significant; for the TXL group, LVSP increased, but LVEDP decreased, and the difference was significant (Table 1).

\section{Myocardial morphological changes of rats}

Cardiac muscle of the CON group was arranged in order; for rats in the DM group, cardiac myocyte showed hypertrophy, myocardial myofibril was deranged, myofilament components reduced, and the gap between was noticeably wider; lesions of the TXL group were milder than that in the DM group (Figure 1).

\section{Effects of tongxinluo on the myocardial ultrastructure}

Under electron microscope, it can be seen that the capillary vessel basilar membrane of the cardiac muscle of rats in CON group was continuous and complete; the capillary vessel basilar membrane of the cardiac muscle of rats in DM group was significantly thickened, endothelial cells were significantly swollen and broke into the lumen in a "hump" like manner; thickening of the capillary vessel basilar membrane of the cardiac muscle of rats in TXL group was significantly reduced. The capillary basement membrane thickness of the normal control group (115 \pm $10 \mathrm{~nm})<$ that of the TXL group $(148 \pm 22 \mathrm{~nm})<$ that of the DM group $(247 \pm 28 \mathrm{~nm}$ ) (all $\mathrm{P}<0.01)$ (Figure 2).

\section{DISCUSSION}

Diabetic cardiomyopathy (DCM) is a specific condition which is primarily manifested by left ventricular remodeling and diastolic and/or systolic dysfunction (Shankar et al., 2007). The primary pathological changes of DCM include the hypertrophy and apoptosis of myocardial cells, myocardial fibrosis, and myocardial microvascular extensive endometrial lesions (Okoshi et al., 2007), which lead to a decrease in myocardial compliance. These pathological changes can be fundamentally ascribed to hyperglycemia-caused glucose and lipid disorders. As an independent risk factor, hyperglycemia can directly cause myocardial damage and ultimately, lead to DCM (Nielsen et al., 2002).

DCM is an independent cardiomyopathy, has been recognized as a unique cardiac complication of diabetes mellitus (DM), and is closely related to the high incidence and high mortality of cardiovascular disease in diabetic patients. Early DCM show no obvious clinical manifestations, ventricular diastolic dysfunction can be found by color Doppler echocardiography, ventricular systolic dysfunction will gradually occur with the progress of the disease until heart failure. More than $90 \%$ of clinical diabetic patients were with type 2 diabetes (T2DM), and recent studies have shown that, in type 2 diabetes patients, in whom blood glucose was well controlled, $60 \%$ of the individuals still had diastolic dysfunction, thus study on CM is of important practical significance (Jayasankar et al., 2004).

Battiprolu et al. (2010) found that chronic hyperglycemia results in glucose toxicity, causing damages to cardioblasts, cardiac muscle fibers, and vascular 


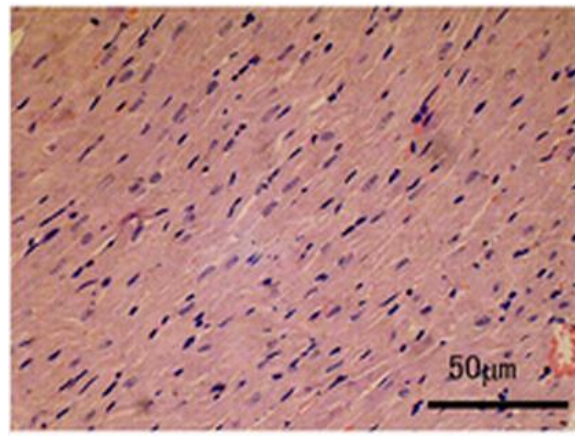

a1

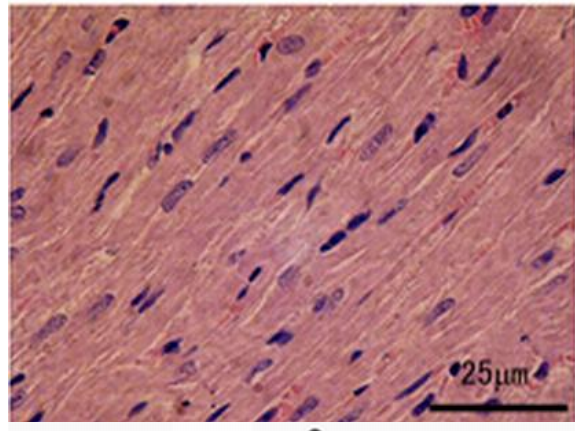

a2

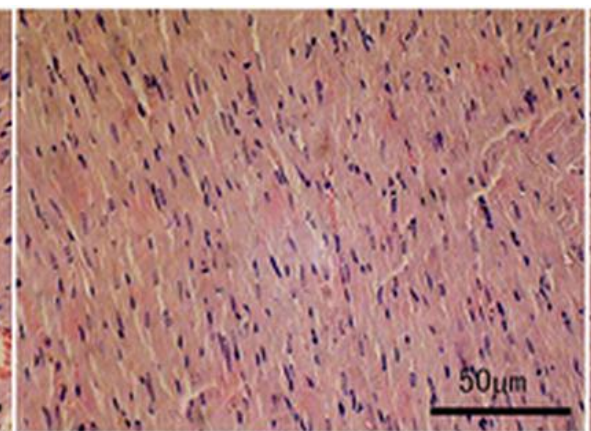

b1

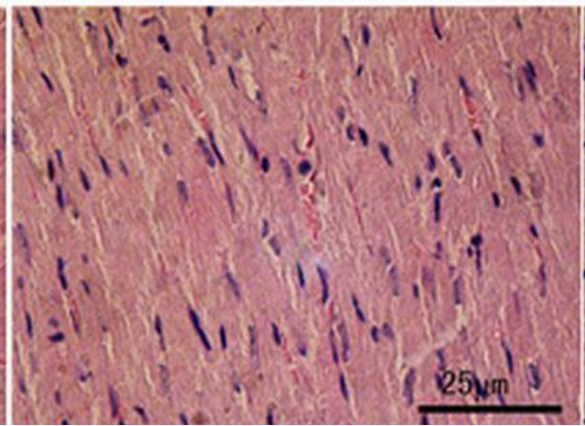

b2

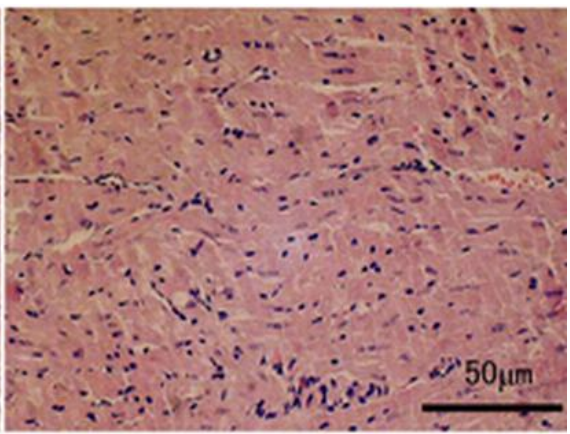

c1

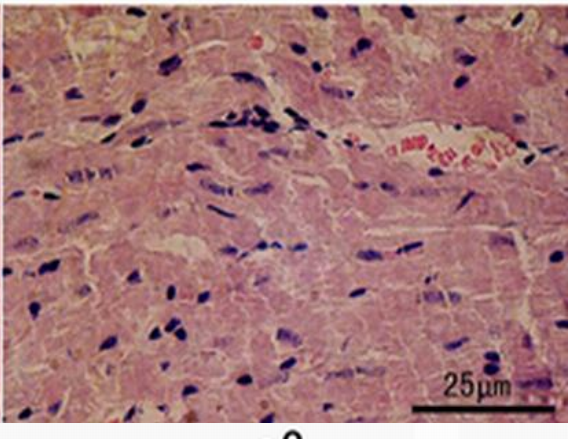

c2

Figure 1. Myocardial morphology changes of rats in each group under light microscope. A1: HE stained CON group (200x); A2: HE stained CON group (400x); B1: HE stained TXL group (200x); B2: HE stained TXL group (400x); C1: HE stained DM group (200x); C2: HE stained DM group (400x).
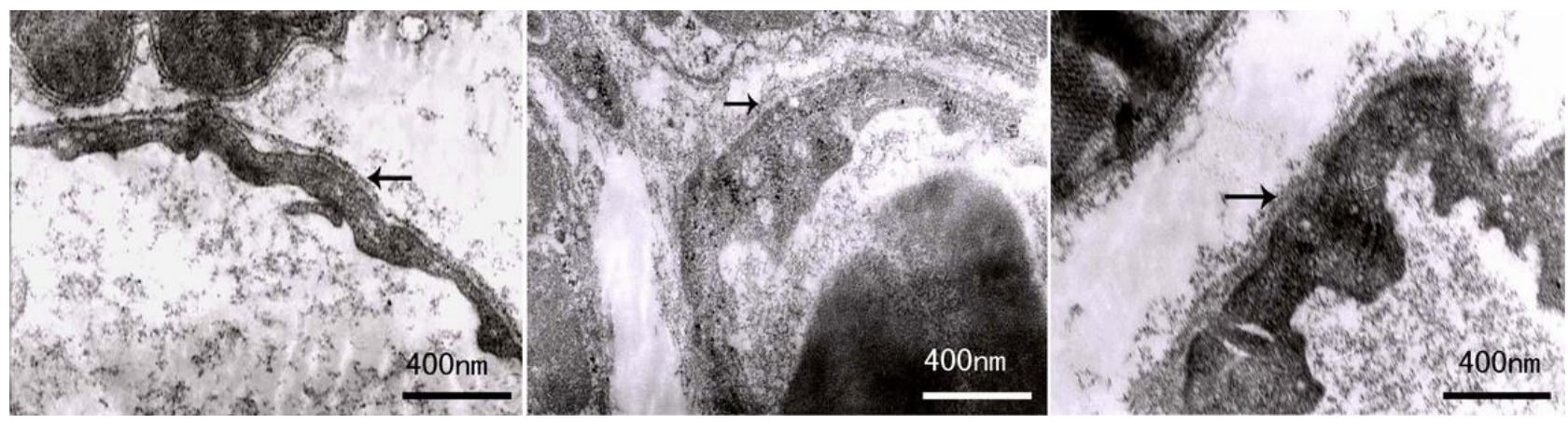

Figure 2. Effects of Tongxinluo on the myocardial ultrastructure of STZ diabetic rats. A: electronic microscope CON group (20000x); B: electronic microscope TXL group (20000x); C: electronic microscope DM group (20000x). Note: $\rightarrow$ marked in electron microscopic observation refers to the capillary vessel basilar membrane.

endothelial cells. Wang et al. (2006) reported that terminal glycosylation end products (AGEs) are one of the mechanisms underlying DCM and that they lead to myocardial fibrosis and cardiac dysfunction.

Diabetic cardiomyopathy is one of the main reasons leading to the death of diabetic patients, therefore, to slow down the pathological process and to improve the prognosis are of important clinical significance in reducing mortality. In this experiment, STZ induced diabetic rat models were established, Tongxinluo was applied for intervention treatment, and was aimed to observe the structural and pathological changes in cardiac muscle and changes in cardiac function in diabetic rats as well as the effect of Tongxinluo on diabetic cardiomyopathy.

\section{Effect of tongxinluo on the cardiac function of rats with STZ-induced diabetes}

DCM triggered by a metabolic disorder can cause myocardial cytological changes, and thus subclinical heart dysfunction will occur and later progress to small 
vessel disease of myocardial muscle, microcirculation disorder and cardiac autonomic neuropathy, and eventually lead to cardiac dysfunction (Shama and Mcneill, 2006). An early manifestation of diabetic cardiomyopathy is decline of left ventricular diastolic function (Fischer et al., 2003). In DM patients, the accumulation of myocardial collagen and deposition of excessive advanced glycation end products (AGE) will result in decrease of left ventricle elasticity and diastolic dysfunction (Asif et al., 2000). Cardiac Doppler examina- tion revealed that, except for type 2 diabetes patients associated with hypertension and coronary heart disease, approximately $60 \%$ of the patients showed reduction in left ventricular diastolic function (Poirier et al., 2001). Studies by Karamitsos et al. (2007) found that, before the occurrence of ventricular systolic dysfunction in diabetic patients, left and right ventricular diastolic dysfunction has already occurred, and this change may be related to interventricular interdependence and the uniform and consistent action of diabetes on the two ventricles.

The results in this experiment suggest that, after injection of STZ, blood glucose was significantly increased, rats showed diabetic symptoms such as polyuria, polydipsia, polyphagia. At eight weeks of course, rats in the DM group showed both cardiac diastolic and systolic function abnormalities, which were manifested as more obvious diastolic dysfunction, and -dp/dt max were significantly reduced; $\pm \mathrm{dp} / \mathrm{dt}$ max in the TXL group was significantly increased, indicating that Tongxinluo improved the diastolic dysfunction of diabetic rat hearts. As reported in literature, changes of cardiomyocytes cardiac function in diabetic rats induced by STZ occurred within 6 to 14 weeks (Thompson, 1988).

This is consistent with our observations. Recently, it is found that diabetic left ventricular diastolic dysfunction is related to the duration of diabetes and hyperglycemia (Henry et al., 2004). Noninsulin-dependent diabetes, hypertension, microalbuminuria or proteinuria, cardiovascular events, and ramipril (DIABHYCAR) studied and observed congestive heart failure in diabetic patients over 50 years old, and found that the basic glycosylated hemoglobin $(\mathrm{GHb})$ level is a predictor of the further development of congestive heart failure (Vanr et al., 2003).

In patients with Type 1 diabetes accompanied by interventricular septal thickening and left ventricular mass increase, with reduction in $\mathrm{GHb}$, interventricular septal thickness and left ventricular mass can be recovered to a certain extent (Aepfelbacher et al., 2004). Studies by Iribarren et al. (2001) have shown that, the degree of impaired diastolic function is related to glycosylated hemoglobin, and the main mechanism is that, advanced glycation end products combined with collagen macromolecules and gathered, which stimulates the release of fibroblast growth factors, resulting in the increase of inflammatory reactions of myocardial cells. It is also reported that, the increased thickness of ventricu- lar in patients with type 1 diabetes can be partially reversed one year later by strictly controlling glucose, which relieved the nosogenesis that blood glucose has been involved in diabetic cardiomyopathy (Grandi et al., 2006).

\section{Effect of tongxinluo on the cardiac function structure of rats with STZ-induced diabetes}

A large number of studies have found that, high blood glucose can lead to myocardial hypertrophy and myocardial fibrosis (Narasimman et al., 2005). Diabetic cardiomyopathy is manifested as deposition of positive materials of myocardial microvascular periodic acid-Schiff reaction (PAS), thickening of the vascular basilar membrane, perivascular fibrous tissue hyperplasia, occlusion and dilatation of myocardial microvascular stenosis (Takai and Miyazaki, 2002). Microvascular disease is one of the important reasons for development of diabetic cardiomyopathy. Recent studies have found that, myocardial collagen deposition and myocardial fibrosis may be an important factor inducing diabetic cardiomyopathy (Way et al., 2002), and its pathophysiological changes are of its own uniqueness.

By continuous observation of the diabetic model animals, it is found that, myocardial fiber hypertrophy, deposition of Periodic acid-Schiff (PAS) positive material among myocardial fibers, focal necrosis of myocardial fibers, fatty degeneratiaon of myocardial cells, thickening of basilar membrane of myocardial capillary vessels, endothelial cell hyperplasia accompanied by mamillary prominency, subendothelial fibrous tissue proliferation, deposition of PAS positive material within the vessel wall, transparent thrombosis within endovascular lumen and vascular stenosis or even occlusion was observed. Travelling disorder of cardiac myofibril can be observed under electron microscope, with different directions of bending, forming several small branches, muscle sections of varying lengths, cystic dilatation of the sarcoplasmic reticulum, myofilament dissolution, formation of myeloid bodies, swelling, limited increase and dissolution of mitochondria deposition of glycogen granules, and significant thickening of capillary vessel basilar membrane.

It was reported in literature that, at 8 weeks after induction with STZ, cardiomyocytes of diabetic rats have shown typical pathological changes of diabetic cardiomyopathy, such as cardiac hypertrophy, hyperplasia, interstitial edema and ultrastructural changes (Zhu et al., 1993). It is observed in this experiment that, under light microscope, cardiac cell hypertrophy, dearrangement of cardiac myofibrils, reduction of myofilament composition, significant widening of interstice can be observed in rats of DM group; significant thickening of the capillary vessel basilar membrane of the cardiac muscle, significant swelling of endothelial cells and their breaking into the 
lumen in a "hump" like manner can be observed in rats in the DM group under electron microscope.

In TXL group, dearrangement of cardiac myofibrils, widening of interstice, thickening of the capillary vessel basilar membrane of the cardiac muscle and swelling of endothelial cells was significantly reduced as compared with that of rats in DM group, therefore, it can be concluded that, Tongxinluo has certain protection effect for cardiac muscle of diabetic rats. For the mechanism of protection function of Tongxinluo for heart, it is believed by clinical studies that, it may mediate the protection effect by increasing nitrogen monoxide (NO) level, to inhibit serum endothelin (ET). Animal experiments show that, Tongxinluo can reduce myocardial infarct size after ischemia reperfusion, reduce the level of plasma creatine kinase (CK), and thus has protection effect for ischemia and reperfusion cardiac muscle.

Tongxinluo is constituted by leech, scorpion, eupolyphaga, centipede, cicada, ginseng, red peony root and borneol, and is of effect of supplementing qi and activating blood circulation, as well as removing obstruction in channels to relieve pain. Ginseng has the effects of invigoration, benefiting qi, regenerating body fluid, regulating immunity, and so on. Ginseng total saponin promotes the aerobic oxidation of glucose, enhances the activity of succinate dehydrogenase, as well as promotes insulin release to some extent. Red peony roots and Eupolyphaga seu steleophaga can decrease blood fats, guard against arteriosclerosis and platelet aggregation, and improve blood hypercoagulability. Furthermore, they can remove oxygen radicals in tissues, inhibit mitochondrial swelling and lipid peroxidation, as well as protect mitochondrial structures and functions. Leeches, scorpions, Periostracum cicadae, scolopendra, and Borneolum syntheticum can promote blood circulation for removing blood stasis, eliminate inflammation and relieve pains, dredge channels and collaterals, dispel wind, and improve immunity, and so on. This study show that Tongxinluo can significantly improve the heart function of diabetic rats, and can reduce diabetic cardiomyopathy, and its mechanism may be by protecting the microvascular, to prevent diabetes progress to diabetic cardiomyopathy. To date, although some findings concerning the application of Tongxinluo in the prevention and cure of DCM have been obtained, most are obtained from animal experiments. Therefore, to provide wider application prospect for Tongxinluo for this use, a large scale clinical studies remain to be carried out. Furthermore, the specific action mechanisms underlying the effect of Tongxinluo on myocardial preservation remain to be explored.

\section{ACKNOWLEDGEMENT}

This study was supported by Liaoning Provincial Education Department Foundation (project no: L2010284) Liaoning Provincial Science and Technology Department and Foundation (project no: 2009225010-42).

\section{REFERENCES}

Aepfelbacher FC, Yeon SB, Weinrauch LA, D'Elia J, Burger AJ (2004). Improved glycemic control induces regression of left ventricular mass in patients with type 1 diabetes mellitus. Int. J. Cardiol. 94: 47-51.

Asif M, Egan J, Vasan S, Jyothirmayi GN, Masurekar MR, Lopez S, Williams C, Torres RL, Wagle D, Ulrich $P$, Cerami A, Brines $M$, Regan TJ (2000). An advanced glycation endproduct cross link breaker can reverse age-related increases in myocardial stiffness. Proc. Natl. Acad. Sci. U S A. 97: 2809-2813.

Battiprolu PK, Gillette TG, Wang ZV, Lavandero S, Hill JA (2010). Diabetic cardiomyopathy: Mechanisms and Therapeutic Targets. Drug Discov. Today Dismech. 7:e135-e143.

Fischer $M$, Baessler A, Hense HW, Hengstenberg C, Muscholl $M$, Holmer S, Döring A, Broeckel U, Riegger G, Schunkert H (2003). Prevalence of left ventrieular diastolic dysfunction in the community. Results from a Doppler echocardiographic-based survey of a population sample. Eur. Heart J. 24: 320-328.

Grandi AM, Piantanida E, Franzetti I, Bernasconi M, Maresca A, Marnini P, Guasti L, Venco A (2006). Effect of glycemic control oil left ventricular diastolic function in type 1 diabetes mellitus. Am. J. Cardiol. 97: 71-76.

Hayat SA, Patel B, Khattar RS, Malik RA (2004). Diabetic cardiomyopathy: mechanisms, diagnosis and treatment. Clin. Sci. 107: 539-557.

Henry RM, Kamp O, Kostense PJ, Spijkerman AM, Dekker JM, van Eijck R, Nijpels G, Heine RJ, Bouter LM, Stehouwer CD (2004). Left ventricular mass increases with deteriorating glucose tolerance, especially in women: independence of increased arterial stiffness or decreased flow - mediated dilation: the Hoorn study. Diabetes Care. 27: $522-529$

Iribarren C, Karter AJ, Go AS, Ferrara A, Liu JY, Sidney S, Selby JV (2001). Glycemic control and heart failure among adult patients with diabetes. Circulation. 103: 2668-2673.

Jayasankar V, Woo YJ, Bish LT, Pirolli TJ, Berry MF, Burdick J, Bhalla RC, Sharma RV, Gardner TJ, Sweeney HL (2004). Inhibition of matrix metalloproteinase activity by TIMP-1 gene transfer effectively treats ischemic cardiomyopathy. Circulation. I10: 180-186.

Karamitsos TD, Karvounis HI, Dalamanga EG, Papadopoulos CE, Didangellos TP, Karamitsos DT, Parharidis GE, Louridas GE (2007). Early diastolic inpairment of diabetic heart the significance of right ventricle. Int. J. Cardiol. 114: 218-223.

Laakso M (1999). Hyperglycemia and cardiovascular disease in type 2 diabete. Diabetes. 48: 937-942.

Narasimman G, Kenichi W, Ma M, Zhang S, Muslin AJ, Kodama M, Aizawa $Y$ (2005). Inactivation of $14-3-3$ protein exacerbates cardiac hypotrophy and fibrosis through enhanced expression of protein kinase $C \beta 2$ in experimental diabetes. Biol. Pharm. Bull. 28: 957-962.

Nielsen LB, Bartels ED, Bollano E (2002). Over expression of Apo lipoprotein $B$ in the heart impedes cardiac triglyceride accumulation and development or cardiac Dysfunction in diabetic mice. J. Biol. Chem. 277:7014-27020.

Okoshi K, Guimaraes JF, Di Muzio Muzio BP, Fernandes AA, Okoshi MP (2007). Diabetic cardiomyopathy. Arg. Bras. Endocrinal Metabol. 51:160-167.

Poirier P, Bogaty P, Garneau C, Marois L, Dumesnil JG (2001). Diastolic dysfunction in normotensive men with well-controlled type 2 diabetes: importance of maneuvers in echocardiographic screening for preclinical diabetic cardiomyopathy. Diabetes Care. 24:5-10.

Rubler S, Dlugash J, Yuceoglu YZ, Kumral T, Branwood AW, Grishman A (1972). New type of cardiomyopathy associated with diabetic glomerulosclerosis. Am. J. Cardiol. 30:595-602.

Shama V, Mcneill JH (2006). Diabetic cardiomyopathy: where are we 40 years later? Can J. Cardiol. 22:305-308.

Shankar A, Li J, Nieto FJ, Klein BE, Klein R (2007). Association between creative protein level and peripheral arterial disease among US adults without cardiovascular disease, diabetes, or hypertension. Am. Hear J. 54:495-501.

Takai S, Miyazaki M (2002). The Role of Chymase in Vascular 
Proliferation. Drug News Perspect. 15:278-282.

Thompson EW (1988). Structural manifestations of diabetic cardiomyopathy in the rats and its reversal by insulin treatment. Am. J. Anat. 182:270-282.

Vanr L, Gueret P, Lievre M, Chabaud S, Passa P (2003). Development of congestive heart failure in type 2 diabetic patients with microalbuminuria or proteinuria: observations from the DIABHYCAR (type 2 Diabetes, Hypertension, Cardiovascular Events and (Ramipril) study. Diabetes Care. 26:855-860.

Wang J, Song y, Wang Q, Kralik PM, Epstein PN (2006). Causes and characteristics of diabetic cardiomyopathy. Rev. Diabet Stud. 3:108117.
Way KJ, Isshiki K, Suzuma K, Yokota T, Zvagelsky D, Schoen FJ, Sandusky GE, Pechous PA, Vlahos CJ, Wakasaki H, King GL (2002). Expression of connective tissue growth factor is increased in injured myocardium associated with protein kinase $\mathrm{C}$ beta2 activation and diabetes. Diabetes. 51:2709-2718.

Zhu XX, Zhou XP, Zhong XL, Yang XF, Zhong CS, Yu YF (1993). Streptozotocin induced cardiomyopathy in diabetic rats. Chin. Med. J. 106:463-465. 